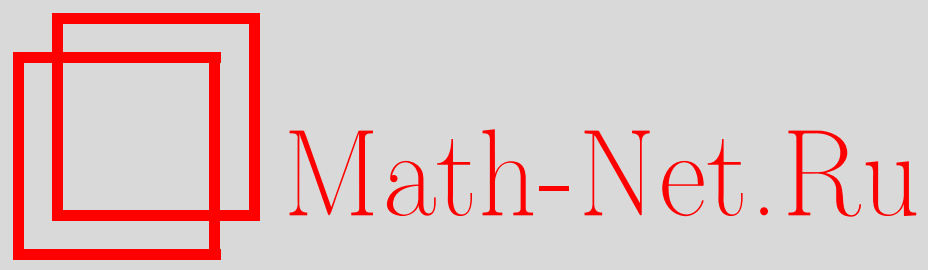

Ю. М. Березанский, Бесконечномерный негауссов анализ и операторы обобщенного сдвига, Функи. анализ и его прил., 1996, том 30, выпуск 4, 61-65

DOI: https://doi.org/10.4213/faa551

Использование Общероссийского математического портала MathNet.Ru подразумевает, что вы прочитали и согласны с пользовательским соглашением

http://www . mathnet.ru/rus/agreement

Параметры загрузки:

IP : 54.198 .55 .26

26 апреля 2023 г., 14:44:22

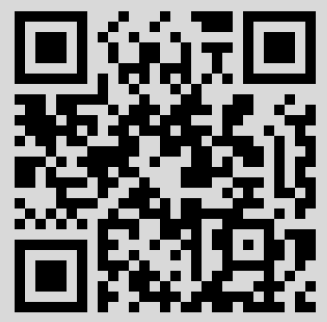


УдК 517.515

\title{
Бесконечномерный негауссов анализ и операторы обобщенного сдвига
}

\author{
(C) 1996. Ю. М. БЕРЕЗАНСКИй
}

Гауссов бесконечномерный анализ $[1,2]$ в последнее время обобщался на негауссовы меры, и одно из таких обобщений основано на замене ортогональных разложений на биортогональные $[3,4]$. В [5-7] установлено, что в модельном одномерном случае подход, использованный в $[3,4]$, может быть широко обобщен, если вместо экспонент брать характеры $L_{1}$-гипергруппы, а вместо обычного сдвига - обобщенный. В этой заметке показано, что то же можно сделать и в бесконечномерном случае и получить в общей ситуации результаты, охватывающие результаты работ $[3,4]$. При этом детальная теория $L_{1}$-гипергрупп с не локально компактным базисом $Q$ (которая пока не построена) сейчас не нужна, достаточно пользоваться операторами обобщенного сдвига $T_{x}(x \in Q)$, удовлетворяющими весьма простым требованиям.

1. Пусть $Q$ - сепарабельное метрическое полное пространство точек $x, y, \ldots$ и $C(Q)$ - линейное пространство всех комплекснозначных локально ограниченных (т. е. ограниченных на каждом шаре из $Q$ ) непрерывных функций на $Q$. Предположим, что в $C(Q)$ задано семейство $T=\left\{T_{x}\right\}_{x \in Q}$ линейных операторов «обобщенного сдвига», обладающих следующими свойствами: (a) $\left(T_{x} f\right)(y)=\left(T_{y} f\right)(x)(x, y \in Q)$ для любой функции $f \in C(Q)$ (коммутативность); (б) существует точка $e \in Q$ (базисная единица), такая, что $T_{e}=\mathrm{id}$; (в) для любых $x, y \in Q$ существует шар $W=W_{x, y} \subset Q$, такой, что для любой функции $f \in C(Q)$ значения $\left(T_{x} f\right)(y)$ не зависят от значений $f(s)$ при $s \in Q \backslash W$ (локальность); (г) если $C(Q) \ni f_{n} \rightarrow f \in C(Q)$ в смысле равномерной сходимости на каждом шаре, то $\left(T_{x} f_{n}\right)(y) \rightarrow\left(T_{x} f\right)(y)$ для любых $x, y \in Q$ (непрерывность).

Характером семейства $T$ будем называть не равную тождественно нулю функцию $\chi \in C(Q)$, удовлетворяющую соотношению $\left(T_{x} \chi\right)(y)=\chi(x) \chi(y)(x, y \in Q)$. Предположим, что для $T$ существует множество характеров $\chi_{\lambda}(x)=\chi(x, \lambda)$, нумерующихся точками $\lambda$ комплексного гильбертова пространства $N_{0}$ и аналитически зависящих от $\lambda$.

Более точно, рассмотрим для любых $p \in \mathbb{N}$ и $n \in \mathbb{Z}_{+}=\{0,1, \ldots\}$ цепочку комплексных гильбертовых вложенных пространств

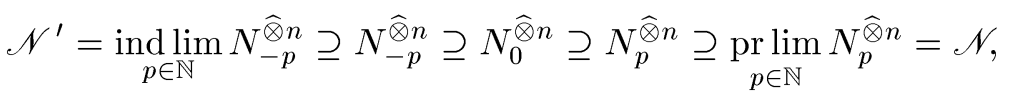

где $\widehat{\otimes}$ обозначает симметрическое тензорное произведение [2] (при $n=0$ пространства из (1) совпадают с $\left.\mathbb{C}^{1}\right)$. Будем предполагать, что эти пространства являются комплексификацией соответствующих вещественных пространств, и пусть $\lambda \mapsto \bar{\lambda}$ - переход к комплексному сопряжению в (1). Вещественное спаривание в (1) будем обозначать через $\langle\cdot, \cdot\rangle$. Таким образом, комплексное спаривание $(\cdot, \cdot)_{N_{0}^{\widehat{\otimes} n}}$ совпадает с $\langle\cdot, \bar{\cdot}\rangle$. Пространство $\mathscr{N}$ предполагается ядерным, а вложения $N_{1} \hookrightarrow N_{0}, N_{2} \hookrightarrow N_{1}$ и $N_{3} \hookrightarrow N_{2}$ - вложениями Гильберта-Шмидта. 
Итак, предположим, что для любой точки $x \in Q$ характер $\chi(x, \lambda)$ является аналитической функцией переменной $\lambda \in N_{0}$ в некоторой общей для $x$ окрестности $B_{0}$; при этом функции $\chi(\cdot, \lambda)$ равномерно относительно $\lambda \in B_{0}$ ограничены на каждом шаре из $Q$. Аналитичность приводит к существованию для любой точки $x \in Q$ равномерно сходяшегося при некотором $R_{1}>0$ разложения

$$
\chi(x, \lambda)=\sum_{n=0}^{\infty} \frac{1}{n !}\left\langle\lambda^{\otimes n}, \chi_{n}(x)\right\rangle, \quad \lambda \in B_{1}=\left\{\lambda \in N_{1} \mid\|\lambda\|_{N_{1}} \leqslant R_{1}\right\},
$$

с коэффициентами $Q \ni x \mapsto \chi_{n}(x) \in N_{-1}^{\widehat{\otimes} n} \subseteq N_{-2}^{\widehat{\otimes} n}-$ характерами Дельсарта. Нетрудно показать, что они слабо непрерывны и $\left\|\chi_{n}(\cdot)\right\|_{N_{-2}^{\widehat{\otimes}_{n}}}$ локально ограничена для любого $n$. Действие $T_{x}$ переносится на $\chi_{n}(\cdot)$ и легко подсчитывается.

2. Пусть $\rho$ - борелевская вероятностная мера на $Q$, положительная на открытых множествах. Ниже строится теория обобщенных функщий переменной $x \in Q$ с комплексным спариванием $\langle\langle\cdot, \cdot\rangle$, задающимся скалярным произведением пространства $H_{0}=L_{2}(Q, d \rho(x))$. В этом разделе рассматриваются «пространства Дельсарта», построенные по $\chi_{n}$.

Предположим, что семейство $T$ и мера $\rho$ таковы, что для некоторого $C>0$

$$
\|\| \chi_{n}(\cdot)\left\|_{N_{-2}^{\widehat{\otimes} n}}\right\|_{H_{0}} \leqslant C^{n} n ! \quad\left(n \in \mathbb{Z}_{+}\right)
$$

и элементарные функции $Q \ni x \mapsto\left\langle a^{n}, \chi_{n}(x)\right\rangle \in \mathbb{C}^{1}$ при $a^{n} \in N_{2}^{\widehat{\otimes} n}$ (входящие в силу $(3)$ в $H_{0}$ ) линейно независимы и их линейная оболочка плотна в $H_{0}$. Введем обозначение $\mathbb{N}_{k}=\{k, k+1, \ldots\}, k \in \mathbb{N}$.

Зафиксируем $p \in \mathbb{N}_{2}, q \in \mathbb{N}$ и достаточно большое $K>1$ и рассмотрим гильбертово пространство

$$
\begin{aligned}
H^{\chi}(p, q)=\left\{\varphi(x)=\sum_{n=0}^{\infty}\left\langle a^{n}, \chi_{n}(x)\right\rangle, a^{n} \in N_{p}^{\widehat{\otimes} n} \mid\right. \\
\left.\|\varphi\|_{H^{\chi}(p, q)}^{2}=\sum_{n=0}^{\infty}\left\|a^{n}\right\|_{N_{p}^{\widehat{\otimes} n}}^{2}(n !)^{2} K^{q n}<\infty\right\}
\end{aligned}
$$

с соответствующим скалярным произведением. Таким образом, $H^{\chi}(p, q)$ изометрично пространству Фока $\mathscr{F}\left(N_{p, \text { Re }}\right)$, взвешенному множителями $(n !)^{2} K^{q n}$.

Пространство $H^{\chi}(p, q)$ можно рассматривать как позитивное относительно нулевого $H_{0}$, и пусть $H^{\chi}(-p,-q)$ - соответствующее сопряженное (негативное) пространство обобщенных функций. Для их описания введем понятие кохарактера Дельсарта.

Пусть $\alpha^{m} \in N_{-p}^{\widehat{\otimes} m}$, где $p \in \mathbb{N}_{2}, m \in \mathbb{Z}_{+}$. Образуем операцию «уничтожения» $\partial\left(\alpha^{m}\right)$ с коэффициентом $\alpha^{m}$, полагая

$$
\partial\left(\alpha^{m}\right)\left\langle a^{n}, \chi_{n}(x)\right\rangle= \begin{cases}\frac{n !}{(n-m) !}\left\langle a^{n}, \alpha^{m} \widehat{\otimes} \chi_{n-m}(x)\right\rangle, & m \leqslant n, \\ 0, & m>n,\end{cases}
$$

для любого $n \in \mathbb{Z}_{+}$и любого $a^{n} \in N_{p}^{\widehat{\otimes} n}$.

Утверждается, что для любых $p \in \mathbb{N}_{2}, q \in \mathbb{N}$ оператор $\partial\left(\alpha^{m}\right)$ действует непрерывно в пространстве $H^{\chi}(p, q)$ и, следовательно, его сопряженный $\partial^{+}\left(\alpha^{m}\right)$ (относительно $\left.H_{0}\right)$ - в $H^{\chi}(-p,-q)$. Кохарактер Дельсарта $\theta\left(\alpha^{m}\right)$ 
определяется равенством $\theta\left(\alpha^{m}\right)=\partial^{+}\left(\overline{\alpha^{m}}\right) \delta_{e} \in H^{\chi}(-p,-q)$, где $\delta_{e}$ есть $\delta$-функция, сосредоточенная в точке $e \in Q$ (она входит в $\left.H^{\chi}(-p,-q)\right)$. Cnpaведливо соотношение ортогональности:

$\left\langle\left\langle\theta\left(\alpha^{m}\right),\left\langle a^{n}, \chi_{n}(\cdot)\right\rangle\right\rangle\right\rangle=\delta_{n, m} n !\left\langle\alpha^{n}, \overline{a^{n}}\right\rangle \quad\left(\alpha^{m} \in N_{-p}^{\widehat{\otimes} m}, a^{n} \in N_{p}^{\widehat{\otimes} n}, n, m \in \mathbb{Z}_{+}\right)$.

Теорема 1. Пространства $H^{\chi}(p, q)\left(p \in \mathbb{N}_{2}, q \in \mathbb{N}\right)$ u их сопряженнье образуют следуюшую иепочку пространств:

$$
\begin{aligned}
&\left(\Phi^{\chi}\right)^{\prime}=\operatorname{ind}_{p \in \mathbb{N}_{2}, q \in \mathbb{N}} \lim ^{\chi}(-p,-q) \supseteq H^{\chi}(-p,-q) \\
& \supseteq H_{0} \supseteq H^{\chi}(p, q) \supseteq \operatorname{pr}_{p \in \mathbb{N}_{2}, q \in \mathbb{N}} H^{\chi}(p, q)=\Phi^{\chi},
\end{aligned}
$$

причем $\Phi^{\chi}$ ядерное. Пространство $H^{\chi}(-p,-q)$ имеет следующий вид:

$$
\begin{gathered}
H^{\chi}(-p,-q)=\left\{\xi=\sum_{n=0}^{\infty} \theta\left(\alpha^{n}\right), \alpha^{n} \in N_{-p}^{\widehat{\otimes} n} \mid\right. \\
\left.\|\xi\|_{H^{\chi}(-p,-q)}^{2}=\sum_{n=0}^{\infty}\left\|\alpha^{n}\right\|_{N_{-p}^{\widehat{\otimes} n}}^{2} K^{-q n}<\infty\right\}, \\
\left\langle\langle\xi, \varphi\rangle=(\xi, \varphi)_{H_{0}}=\sum_{n=0}^{\infty}\left\langle\alpha^{n}, \overline{a^{n}}\right\rangle n ! \quad\left(\xi \in H^{\chi}(-p,-q), \varphi \in H^{\chi}(p, q)\right) .\right.
\end{gathered}
$$

Заметим, что множитель $(n !)^{2}$ в (4) определяется мерой $\rho$, допускающей оценку (3). При определенных $T$ и $\rho$ разумны и другие оценки типа $(3)$, влекущие замену $(n !)^{2}$ соответствующим множителем.

3. Для построения «пространств Аппеля» зафиксируем функционал $l \in$ $H^{\chi}(-2,-1)$ и для любого $\lambda \in N_{2}$ рассмотрим функцию $\hat{l}(\lambda)=l(\overline{\chi(\cdot, \lambda)})$. При малой $\|\lambda\|_{N_{2}}$ она определена и является аналитической. Пусть $l$ таков, что $\hat{l}(0) \neq 0$; тогда для любой точки $x \in Q$ функция $\sigma(x, \lambda)=\chi(x, \lambda) \hat{l}^{-1}(\lambda)$ аналитична в окрестности нуля, и поэтому справедливо разложение типа (2) при некотором $R_{2}>0$ :

$$
\sigma(x, \lambda)=\sum_{n=0}^{\infty} \frac{1}{n !}\left\langle\lambda^{\otimes n}, P_{n}(x)\right\rangle, \quad \lambda \in B_{2}=\left\{\lambda \in N_{2} \mid\|\lambda\|_{N_{2}} \leqslant R_{2}\right\},
$$

с коэффициентами $Q \ni x \mapsto P_{n}(x) \in N_{-2}^{\widehat{\otimes} n} \subseteq N_{-3}^{\widehat{\otimes} n}-$ характерами Аппеля.

Характеры Аппеля выражаются через характеры Дельсарта при помощи линейного обратимого преобразования. Для них автоматически справедлива оценка типа (3) с заменой $N_{-2}$ на $N_{-3}$ и линейная независимость и плотность в $H_{0}$ линейной оболочки элементарньх функиий $\left\langle a^{n}, P_{n}(x)\right\rangle\left(a^{n} \in N_{3}^{\widehat{\otimes} n}\right.$, $\left.n \in \mathbb{Z}_{+}\right)$. Построим пространство $H^{P}(p, q)\left(p \in \mathbb{N}_{3}, q \in \mathbb{N}\right)$ аналогично (4), но с заменой $\chi_{n}(x)$ на $P_{n}(x) ; H^{P}(-p,-q)$ - сопряженное к нему пространство. Вместо (6) имеем цепочку

$$
\begin{aligned}
\left(\Phi^{P}\right)^{\prime}=\operatorname{ind}_{p \in \mathbb{N}_{3}, q \in \mathbb{N}} \lim ^{P}(-p,-q) & \supseteq H^{P}(-p,-q) \\
& \supseteq H_{0} \supseteq H^{P}(p, q) \supseteq \underset{p \in \mathbb{N}_{3}, q \in \mathbb{N}}{\operatorname{pr} \lim _{q} H^{P}(p, q)=\Phi^{P} .}
\end{aligned}
$$


Tеорема 2. Cуществует $t \in \mathbb{Z}_{+}$, такое, ито для любьх $p \in \mathbb{N}_{3}, q \in \mathbb{N}$ имеют место плотнье непрерьвныле вложения $H^{\chi}(p, q+t) \hookrightarrow H^{P}(p, q)$, $H^{P}(p, q+t) \hookrightarrow H^{\chi}(p, q)$. Таким образом, $\Phi^{\chi}=\Phi^{P}=\Phi,\left(\Phi^{\chi}\right)^{\prime}=\left(\Phi^{P}\right)^{\prime}=\Phi^{\prime}$.

Результаты теоремы 1 сохранлются для чепочки (9) со следуюшими изменениям: вместо $\theta\left(\alpha^{n}\right)$ фигурируют кохарактеры Аппеля $Q\left(\alpha^{n}\right)$, определяюшиесл, как и $\theta$, но с заменой $\delta_{e}$ на $l ;$ в $(7) p \in \mathbb{N}_{3}, q \in \mathbb{N}_{1+t}$. Для $Q\left(\alpha^{m}\right)$ $u\left\langle a^{n}, P_{n}(x)\right\rangle$ справедливо прежнее соотношение ортогональности (5) при $p \in \mathbb{N}_{3}$.

Заметим, что в случае «классических» характеров Аппеля, отвечающих функционалу $l=1$ (т.е. $l(\varphi)=\int_{Q} \overline{\varphi(x)} d \rho(x)$ ), во второй части теоремы 2 можно брать $p \in \mathbb{N}_{2}$ и $q \in \mathbb{N}$.

4. Как и в случае обычного сдвига, сейчас могут быть введены интегральные преобразования $C, S$ и $T$. Так, на некоторых запасах основных и обобщенных функций $\varphi \in \Phi, \xi \in \Phi^{\prime}$ определим эти операторы формулами

$$
(C \varphi)(x)=l\left(\overline{\left(T_{x} \varphi\right)(\cdot)}\right), \quad(S \xi)(\lambda)=\left\langle\left\langle\xi, k_{S}(\lambda, \cdot)\right\rangle\right\rangle, \quad(T \xi)(\lambda)=\left\langle\left\langle\xi, k_{T}(\lambda, \cdot)\right\rangle,\right.
$$

где $k_{S}(\lambda, x)=\sigma(x, \bar{\lambda}), k_{T}(\lambda, x)=\chi(x, \bar{\lambda}), x \in Q, \lambda \in N_{3},\|\lambda\|_{N_{3}}<R_{3}$ и $R_{3}>0$ достаточно мало.

Теорема 3. Оператор $C$ является унитарныл и переводит $H^{P}(p, q)$ в $H^{\chi}(p, q) ;$ при этом $\left(C\left\langle a^{n}, P_{n}(\cdot)\right\rangle\right)(x)=\left\langle a^{n}, \chi_{n}(x)\right\rangle$ для любых $a^{n} \in N_{p}^{\widehat{\otimes} n}$, $x \in Q\left(n \in \mathbb{Z}_{+}, p \in \mathbb{N}_{3}, q \in \mathbb{N}_{1+t}\right) ;$ если $C^{+}$- сопряженный $\kappa$ нему операmop, mo $C^{+} \theta\left(\alpha^{n}\right)=Q\left(\alpha^{n}\right)\left(\alpha^{n} \in N_{-p}^{\widehat{\otimes} n}\right)$.

Oператор $S$ определен на всем пространстве $\Phi^{\prime}=\left(\Phi^{P}\right)^{\prime}$ и переводит его в некоторое пространство $\operatorname{Hol}_{0}(\mathscr{N})$ ростков аналитических функций на проективном пределе $\mathscr{N}\left(\right.$ см. (1); при этом $S Q\left(\alpha^{n}\right)=\left\langle\alpha^{n}, \lambda^{\otimes n}\right\rangle\left(\alpha^{n} \in N_{-p}^{\widehat{\otimes} n}\right.$, $\left.n \in \mathbb{Z}_{+}, p \in \mathbb{N}_{3}\right)$.

Оператор $T$ также определен на всем $\Phi^{\prime}=\left(\Phi^{\chi}\right)^{\prime}$ и переводит его в прежнее $\left.\mathrm{Hol}_{0}(\mathscr{N})\right)$; при этом выше $Q\left(\alpha^{n}\right)$ нужно заменить на $\theta\left(\alpha^{n}\right)$.

Отметим, что пространство $\operatorname{Hol}_{0}(\mathscr{N})$ (которое легко описывается) является алгеброй относительно обычных операчий. Поэтому обратные отображения $S^{-1}$ и $T^{-1}$ превращают $\Phi^{\prime}$ в алгебры с различными умножениями «S-Вика» и «T-Вика»: $\diamond_{S}$ и $\diamond_{T}$.

5. Примеры. (а) (классическая ситуация) $Q$ совпадает с вещественным гильбертовым пространством $X,\left(T_{x} f\right)(y)=f(y+x)(x, y \in X), e=0$, $N_{0}=X_{\text {с }}$ (комплексификация $\left.X\right), \chi(x, \lambda)=\exp (\lambda, x)_{X_{\mathrm{c}}}, \chi_{n}(x)=x^{\otimes n}$.

(б) $Q$ равно пространству пар $\boldsymbol{x}=\{x,-x\}(x \in X),\left(T_{\boldsymbol{x}} f\right)(\boldsymbol{y})=\frac{1}{2}(f(y+x)+$ $f(y-x))(x \in \boldsymbol{x}, y \in \boldsymbol{y}), e=0, N_{0}=X_{\mathrm{c}}, \chi(\boldsymbol{x}, \lambda)=\cos (\lambda, x)_{X_{\mathrm{c}}}, \chi_{2 m}(\boldsymbol{x})=$ $(-1)^{m} x^{\otimes 2 m}, \chi_{2 m+1}=0\left(m \in \mathbb{Z}_{+}\right)$.

(в) Пусть $Q_{\nu}, T^{(\nu)}, \chi^{(\nu)}$ - пространство и соответствующие семейство операторов обобщенного сдвига $T_{x_{\nu}}^{(\nu)}$ и характер $(\nu=1, \ldots, m)$. Положим $Q=Q_{1} \times$ $\cdots \times Q_{m} \ni x=\left\{x_{1}, \ldots, x_{m}\right\}$ и $\left(T_{x} f\right)(y)=\left(\left(T_{x_{1}}^{(1)} \otimes \cdots \otimes T_{x_{m}}^{(m)}\right) f\right)\left(y_{1}, \ldots, y_{m}\right)$ $(x, y \in Q)$. Операторы $T_{x}$ определяют обобщенный сдвиг на $Q$, и $\chi(x, \lambda)=$ $\chi^{(1)}\left(x_{1}, \lambda_{1}\right) \cdots \chi^{(m)}\left(x_{m}, \lambda_{m}\right)\left(\lambda=\left\{\lambda_{1}, \ldots, \lambda_{m}\right\}\right)$ будет его характером. Комбинируя таким образом (а), (б) с одномерными примерами из [5-7], получим ряд новых бесконечномерных примеров. 
6. Как и в модельной одномерной ситуации [5-7], могут рассматриваться операторы обобщенного сдвига Тейлора-Дельсарта, порожденные семейством операторов $\mathscr{L}\left(\alpha^{m}\right)$, таких, что $\left(\mathscr{L}\left(\alpha^{m}\right) \chi(\cdot, \lambda)\right)(x)=\left\langle\alpha^{m}, \lambda^{\otimes m}\right\rangle \chi(x, \lambda)$ для любых $\alpha^{m} \in N_{-p}^{\widehat{\otimes} m}\left(x \in Q, \lambda \in N_{p}, m, p \in \mathbb{N}\right)$. Сейчас $\partial\left(\alpha^{m}\right)=\mathscr{L}\left(\alpha^{m}\right)$.

\section{ЛиТЕРАТУРА}

1. Hida T., Kuo H.-H., Potthoff J., Streit L. White Noise. Infinite Dimensional Calculus. Kluwer Acad. Publ., Dordrecht-Boston-London, 1993. 2. Березанский Ю. М., Кондратьев Ю. Г. Спектральные методы в бесконечномерном анализе. Наукова думка, Киев, 1988. 3. Albeverio S., Daletsky Yu. L., Kondratiev Yu. G., Streit L. NonGaussian Infinite Dimensional Analysis. Bielefeld, Preprint BiBoS, 1994. 4. Kondratiev Yu. G., Streit L., Westerkamp W., Yan J.-A. Generalized Functions in Infinite Dimensional Analysis. IIAS Preprint, 1995. 5. Березанский Ю. М., Кондратьев Ю. Г. Функц. анализ и его прил., 29, вып. 3, 51-55 (1995). 6. Berezansky Yu. M. Rep. Math. Phys, 38, No. 3 (1996). 7. Berezansky Yu. M., Kondratiev Yu. G. Methods Funct. Anal. Topology, 2, No. 2 (1996).

Институт математики НАНУ, Киев, Украина

Университет MCS, Люблин, Польша

Поступило в редакцию 13 июня 1996 г.

УдК 513.88

\section{О крайних точках выпуклых компактов в гильбертовом кубе}

(c) 1996. Е. М. БРонштЕЙн

Сформулируем сначала основные результаты работы.

Теорема 1 . Пусть $M$ является $G_{\delta}$-подмножеством метрического компакта $K$. Существует непрерывное отображение $\varepsilon: K \rightarrow H$, сужение которого на $M$ есть топологическое вложение, такое, что $\operatorname{ext} \operatorname{conv} \varepsilon(K)=$ $\varepsilon(M)$.

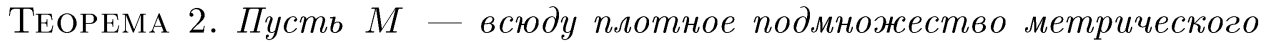
компакта $K$, причем множество $K \backslash M$ слабо счетномерно. Существует топологическое вложение $\varepsilon: K \rightarrow H$, такое, ито $\operatorname{ext} \operatorname{conv} \varepsilon(K)=\varepsilon(M)$.

Здесь conv - замкнутая выпуклая оболочка, ext - множество крайних точек, а $H$ - гильбертов куб. Определение слабо счетномерного компакта см. в [1]. Эти результаты представляют интерес в связи с теоремами Шоке о представлении, которые широко применяются в теории потенциала, теории аппроксимации и др.

Близкие задачи рассматривались в [2-5]; в настоящей работе усилены результаты работ [6-9].

^ Работа поддержана Российским фондом фундаментальных исследований (проект 9401-01286). 